\title{
Does 3T Fetal MRI Improve Image Resolution of Normal Brain Structures between 20 and 24 Weeks' Gestational Age?
}

\author{
(D) G. Priego, (D) N.J. Barrowman, (D). Hurteau-Miller, and (DE. Miller
}

\begin{abstract}
BACKGROUND AND PURPOSE: Stronger magnetic fields have the potential to improve fetal image resolution. Our objective was to detect whether there was better anatomic resolution of brain structures in fetuses imaged with a 3T magnet compared with a 1.5T magnet.
\end{abstract}

MATERIALS AND METHODS: Multiple cerebral and facial anatomic structures were retrospectively assessed in 28 fetal MR imaging scans with normal findings (12 at 3T and 16 at 1.5T) with a 0-3 grading score. Fetuses were assessed during the second trimesters (gestational age, 20-24 weeks). The association between the quality ratings and magnetic field strengths (1.5T versus 3T) was evaluated by a linear mixed-effects model. A quantitative assessment of the signal intensity was also performed in the different layers of the developing brain. Comparative log-ratios were calculated across the different layers of the fetal brain.

RESULTS: There was a statistically significant interaction between location and magnetic field strength $(P<.001)$. The cerebral structures of the cerebellum, pons, venous system, semicircular canal, and cochlea showed statistically significant higher values on the $3 T$ magnet. Similarly, statistical significance was also obtained on the quantitative assessment of the multilayer appearance of the brain; the $3 T$ magnet had a median factor of 8.38 higher than the $1.5 \mathrm{~T}$ magnet $(95 \% \mathrm{Cl}, 4.73-14.82)$. Other anatomic structures assessed in the supratentorial compartment of the brain showed higher values on the $3 \mathrm{~T}$ magnet with no statistical significance.

CONCLUSIONS: Both magnets depict cerebral and facial normal anatomic structures; however, our data indicates better anatomic detail on the $3 \mathrm{~T}$ than on the $1.5 \mathrm{~T}$ magnet.

ABBREVIATIONS: feMRI = fetal MR imaging; GA = gestational age; SAR = specific absorption rate

S ince the first fetal MR imaging was performed in $1983,{ }^{1}$ the technique has developed vastly in the past 3 decades with the use of ultrafast sequences, which minimize motion artifacts ${ }^{2}$ and improve the image quality of the mobile fetus. In addition, the higher magnet strength has already demonstrated superior anatomic contrast resolution in neuroradiology and has been an outstanding diagnostic tool. ${ }^{3-6}$

Recent literature has shown an advantage of 3T magnets in fetal body pathology imaging ${ }^{7,8}$ and in fetal brain resolution. ${ }^{9}$

Received January 13, 2017; accepted after revision March 29.

From the Department of Medical Imaging (G.P., J.H.-M., E.M.) and Research Institute (N.J.B.), Children's Hospital of Eastern Ontario, University of Ottawa, Ottawa, Ontario, Canada. Dr Priego is now with Department of Medical Imaging, Queen's Hospital, London, UK.

Please address correspondence to Elka Miller, MD, FRCPC, Children's Hospital of Eastern Ontario, Department of Medical Imaging, 401 Smyth Rd, Ottawa, ON K1H 8L1, Canada; e-mail: emiller@cheo.on.ca

Indicates article with supplemental on-line table.

Indicates article with supplemental on-line photo.

http://dx.doi.org/10.3174/ajnr.A5251
Other advanced techniques such as MR spectroscopy and diffusion tensor imaging have begun to be applied in fetal imaging with promising results. ${ }^{10,11}$ In addition, the use of stronger magnets has the potential to improve the SNR, which can be used to attain higher image quality, or can be traded for increased temporal resolution (decreased acquisition time) or a combination of both. In the case of fetal imaging, in which the fetus is usually very small and highly mobile, this gain can represent a substantial advantage. The gain in SNR can also allow implementation of SNR applications such as parallel imaging with multichannel coils to speed up single-shot MR imaging protocols, to reduce $\mathrm{TE}$, to mitigate susceptibility, and to decrease radiofrequency heating by minimizing the number of pulses.

As could be anticipated, the main challenge when performing fetal MR imaging at 3T was imaging artifacts. With single-shot FSE sequences, the main artifacts were secondary to B1 inhomogeneity, which is noted as diffuse areas of image shading but did not limit diagnostic interpretation. On the other hand, an increase in the SNR can eliminate the image noise or interference at 
1.5T, which results in improvement of the image quality at $3 \mathrm{~T}$. Motion artifacts were not thought to be different between the 2 magnets. The only perceptible difference is that planning between sequences in the $3 \mathrm{~T}$ magnet took a few seconds longer, which might result in fetal motion.

Small centers with only 1 magnet may prefer to choose the higher field magnet for their clinical practice, which must then fulfill all their imaging requirements, including fetal imaging. Reevaluation of the availability of fetal imaging with 3T magnets can be beneficial in this or other specific instances.

In this study, we aimed to compare the normal anatomy of the fetal brain in the second trimester between images acquired on 1.5T and 3T magnets. The normal anatomy reference of the fetal brain is essential for the interpretation of normal and abnormal fetal MR imaging (feMRI) findings. Our objective was to detect whether there was better anatomic resolution of brain and facial structures in fetuses between the gestational ages (GAs) of 20-24 weeks that were imaged with a 3T magnet compared with a $1.5 \mathrm{~T}$ magnet. Improved anatomic resolution has the potential of a more detailed assessment and an accurate diagnosis and prognosis. ${ }^{12}$

\section{MATERIALS AND METHODS Demographic Data}

This retrospective study was approved by the institutional review board of the Children's Hospital of Eastern Ontario, Canada. All referrals for feMRI followed a dedicated fetal neurosonographic study. No written informed consent was obtained at the Medical Imaging Department, but feMRI requisitions are discussed with a multidisciplinary team prior to booking the MR imaging, as per departmental practice. A search of the Medical Imaging Department data base was performed to identify feMRI studies performed with a 3T system (Magnetom Skyra; Siemens, Erlangen, Germany) and a 1.5T MR imaging system (Sigma HD; GE Healthcare, Milwaukee, Wisconsin) from July 2010 to January 2016. Patients were identified by searching for the term "normal fetal brain" in the final report and having a gestational age between 20 and 24 weeks.

A total of 305 feMRIs (51 performed on the $3 \mathrm{~T}$ and 254 performed on the 1.5T MR imaging scanner) were reviewed. Twenty-eight feMRI scans met the criteria for the term "normal fetal brain" in the final report and had a gestational age between 20 and 24 weeks ( 12 on $3 \mathrm{~T}$ and 16 on 1.5T). Patients were excluded if they did not have normal brain MR imaging findings. Indications for the feMRI of the study group included the following: previous abnormal fetus, mild ventriculomegaly, or other fetal malformation not affecting the brain. No oligo- or polyhydramnios was present in the selected study group.

\section{Sequence Selection}

Every sequence of the selected feMRI studies was reviewed by the pediatric radiology fellow (G.P.), who selected the best diagnostic sequences in each plane. The criteria for selection of the sequences included the following: no or minimal motion artifacts and the presence of the 3 standard brain planes. The sagittal plane included the entire corpus callosum and vermis in the same image.
Table 1: Parameters for 1.5T and 3T fetal MRIs

Fetal Sequences

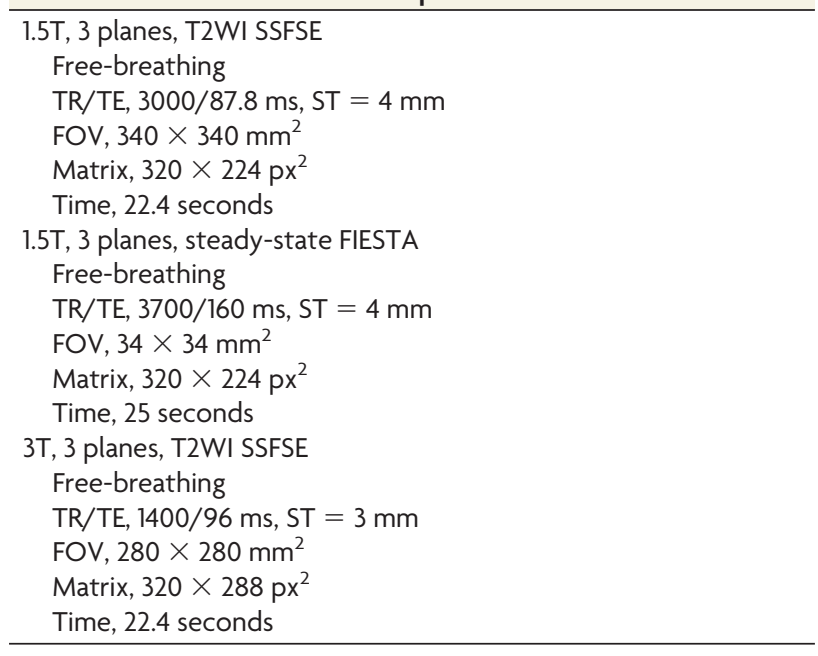

Note:-SSFSE indicates single-shot FSE; px, pixel; ST, section thickness.

Axial and coronal images needed to display a symmetric appearance of the cerebral and cerebellar hemispheres and of the ventricles. In the infratentorial region, the axial plane needed to demonstrate the entire cerebellum and the middle cerebellar peduncles. Angulated or tilted sequences were excluded. Readers were provided with the best preselected sequences. The structures were evaluated with a single-shot FSE sequence obtained with both magnet strengths and with a steady-state acquisition (FIESTA) pulse sequence from the 1.5T magnet.

\section{Imaging Data Acquisition}

Pregnant patients were positioned in the supine or lateral decubitus position. No anesthesia was used. MR images obtained with a 3T MR imaging scanner used a spine matrix coil with 24 elements in the table and a light weight 18-channel phased coil on the surface of the mother. The MR images obtained with a 1.5T MR imaging scanner were acquired with an 8-channel phased array cardiac coil. Imaging parameters for both magnets followed our institutional standard protocol (Table 1).

\section{Image Analysis}

All MR images were evaluated by 2 pediatric neuroradiologists with 16 and 10 years of experience in fetal imaging (E.M., J.H.-M.). Readers were blinded to all clinical information, gestational age, selection of the sequences, and magnet strength. Before starting to read cases, the readers interpreted 4 examinations in consensus to familiarize themselves with the qualitative grading and reach a consensus on the evaluation of the different brain structures and the planes needed for the evaluation. These 4 feMRIs were randomly chosen from both magnet strengths and outside the study group. A mini-atlas was also created for reference by the readers during the independent interpretation. The results of the readers' interpretations were documented in a Research Electronic Data Capture (RedCap; https://catalyst.harvard.edu/services/redcap/) by each investigator.

\section{Qualitative Assessment}

The following structures were evaluated in the coronal and axial images: the multilayered appearance of the brain parenchyma, the 

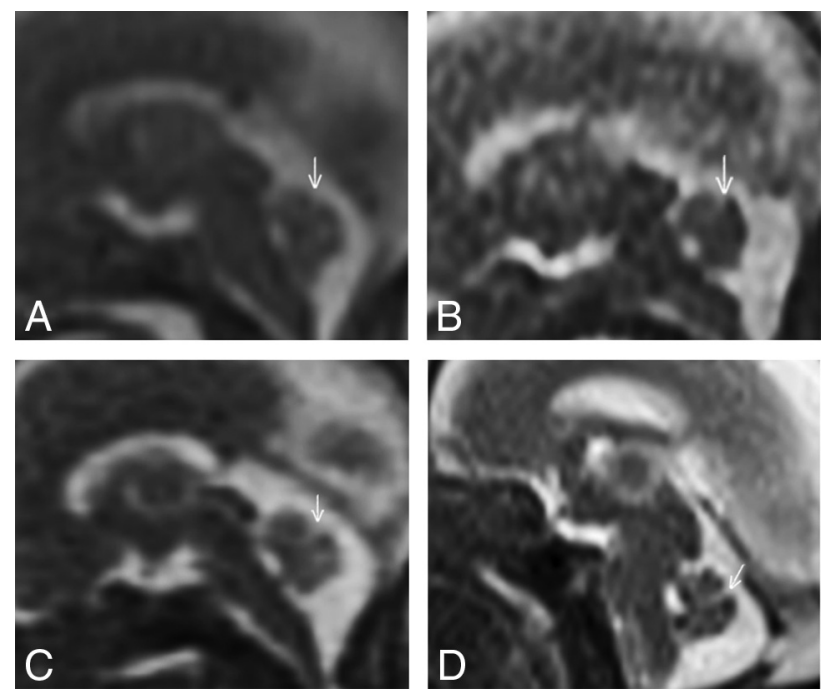

FIG 1. Sagittal T2 midline images of fetuses. Examples provide the qualitative assessment of the primary fissure of the cerebellum (arrows). A, Gestational age (GA), 21 weeks 1 day; 1.5T; score 0: no visible structure. $B, \mathrm{GA}, 21$ weeks; 1.5T; score 1: partially visible structure. C, GA, 22 weeks; 1.5T; score 2: entirely visible structure, but ill-defined contour. D, GA, 20 weeks; 3T; score 3: entirely visible and sharp margins.

septum pellucidum, the cerebellar shape, optic (eye globes, optic nerves, optic chiasm) and ear structures (pinnae, cochlea, semicircular canals), and the choanae. In the sagittal plane, structures were the following: the corpus callosum, vermis and fissures, the belly of the pons, myelination of the midbrain, fastigial point, aqueduct of Sylvius, pituitary stalk, and face profile. In the axial plane, the cerebral peduncles and posterior walls of the optic globe were evaluated. The cerebral venous sinuses were assessed in all 3 planes. The qualitative assessments of the selected structures were rated as follows: 0 represented a structure that was not visible; 1 represented a partially visible structure; 2 represented an entirely visible structure; and 3 represented an excellent resolution of the structure with sharp margins (Fig 1). Approximately 9 months after the initial readings, each reader repeated the image interpretation separated for assessment of the intrarater agreement.

To assess intra- and interrater agreement of the diagnostic quality ratings, the readers used the Cohen weighted $\kappa$. To evaluate the association between diagnostic quality ratings and magnetic field strength, they used a linear mixed-effects model. The model treated fetus as a random effect and reader, location, and magnetic field strength as fixed effects. A thirdorder interaction among location, magnet strength, and gestational age was initially included, together with all lower order interactions and main effects, in particular, an interaction between location and magnet strength. Because the third-order interaction was not statistically significant, it was removed and the model was refitted. At each location, we calculated the estimated average effect of magnetic field strength on diagnostic quality ratings, with a 95\% confidence interval, adjusted for multiple testing. ${ }^{13}$ Two-sided $P$ values $<.05$ were considered statistically significant.

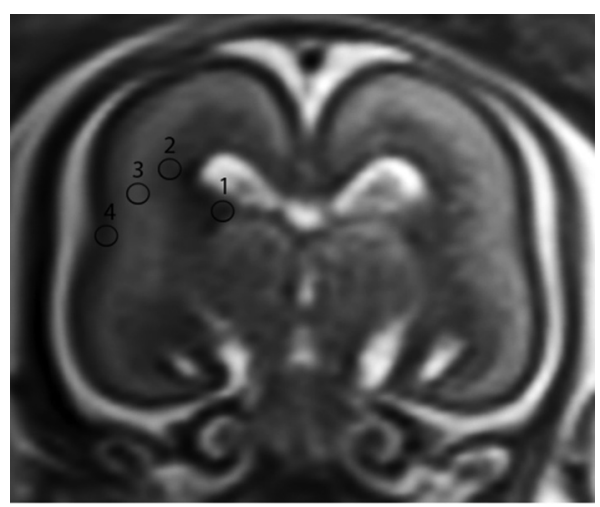

FIG 2. A coronal image was selected from each scan at the level of the third ventricle. One-millimeter ROIs were drawn in the developing brain layers: 1) germinal matrix, 2) periventricular, 3) subplate, and 4) cortical plate. These values were compared with an ROI outside the patient (air). This fetus had a GA of 20 weeks, scanned at 3T.

\section{Quantitative Assessment}

A quantitative assessment by the ROI of the supratentorial brain layers was obtained by a pediatric radiology fellow (G.P.). An ROI of $1 \mathrm{~mm}$ was manually placed on the different layers of the brain (Fig 2): germinal matrix, periventricular layer, subplate layer, and cortical layer. The mean signal intensity for each layer was collected, and a comparative ratio between the different layers and air was calculated. The contrast resolution of each layer was analyzed to compare the different magnet strengths.

The ratios of signal intensity between paired ROIs were compared between the 2 magnetic-field-strength groups. The ratios were $\log$-transformed so that their distributions were approximately normal. A linear mixed-effects model was used to evaluate the association between the log-ratios and magnet strength across different ROIs. The model treated fetus as a random effect, with magnet strength and ROI treated as fixed effects.

\section{RESULTS}

\section{Study Group}

The gestational age range of the 12 fetuses in the group that underwent 3T MR imaging was 20.0-23.9 weeks (median, 21.8 weeks; interquartile range, 21.0-22.2 weeks). The gestational age range of the 16 fetuses in the group that underwent $1.5 \mathrm{~T} \mathrm{MR}$ imaging scans was 20.3-24.7 weeks (median, 21.1 weeks; interquartile range, 20.7-22.7 weeks). The maternal age range was 17.0-39.0 years (median, 32.5 years; interquartile range, 29.534.2 years).

Indications for MR imaging included mild ventriculomegaly $(n=7)$, body malformation $(n=11)$, and abnormality of a fetus in a previous pregnancy $(n=10)$. The cases with mild ventriculomegaly on sonography that were not confirmed on MR imaging were classified as normal feMRI studies. The remaining fetal brain cases were considered normal.

\section{Qualitative Results}

As presented in Table 2, the scores of both readers for all locations demonstrated an increased frequency of score 3 (excellent resolution of the structure with sharp margins) on the $3 \mathrm{~T}$ 
MR imaging scanner $(n=309,56 \%)$ compared with the $1.5 \mathrm{~T}$ MR imaging scanner $(n=108,15 \%)$ and a larger number of score 0 for the $1.5 \mathrm{~T}$ scanner $(n=74,10 \%)$ than for the $3 \mathrm{~T}$ scanner $(n=20,4 \%)$.

In the linear mixed-effects model, the interaction among location, magnet strength, and gestational age was not statistically significant. On removing the interaction and refitting the model, all 2-way interactions were statistically significant, namely location by magnet strength $(P<.001)$, location by gestational age $(P<.001)$, and gestational age by magnet strength $(P=.03)$. Confidence intervals for the interaction of location and magnet strength, adjusted for multiple testing, revealed that 12 structures had a significantly higher diagnostic quality with the $3 \mathrm{~T}$ magnet and none had a significantly lower diagnostic quality. The 3T magnet showed significantly higher diagnostic quality ratings for the following: semicircular canal, cochlea, primary and secondary fissure of the cerebellum, pituitary stalk, fastigial point, pinnae,

Table 2: Frequency of scores within magnet type, including both readers

\begin{tabular}{ccccc}
\hline & \multicolumn{4}{c}{ Magnet Score } \\
\cline { 2 - 5 } & $\mathbf{0}$ & $\mathbf{1}$ & $\mathbf{2}$ & $\mathbf{3}$ \\
\hline $1.5 \mathrm{~T}$ & 74 & 237 & 317 & 108 \\
& $10.1 \%$ & $32.2 \%$ & $43.1 \%$ & $14.7 \%$ \\
$3 \mathrm{~T}$ & 20 & 71 & 152 & 309 \\
& $3.6 \%$ & $12.9 \%$ & $27.5 \%$ & $56.0 \%$ \\
\hline
\end{tabular}

belly of the pons, optic chiasm, and the straight, transverse, and superior sagittal venous sinuses (On-line Table 1). The largest statistically significant difference was for the semicircular canal for which it was estimated that the $3 \mathrm{~T}$ magnet yielded 1.6 points in higher diagnostic quality ratings ( $95 \% \mathrm{CI}, 1.0-2.2)$. Other structures had higher values on the $3 \mathrm{~T}$ scanner than on the $1.5 \mathrm{~T}$ scanner, but with no statistical significance: septum pellucidum, choanae, optic nerves, face, corpus callosum, globes, cerebellar shape, cerebral peduncles, posterior myelination of the pons, and the multilayer appearance of the brain. The only structure that did not show better values on $3 \mathrm{~T}$ was the aqueduct of Sylvius (Fig 3).

The Cohen weighted $\kappa$ for the 2 sets of readings by reader 1 was 0.75 ; for reader 2 , it was 0.76 . The Cohen weighted $\kappa$ between readers was 0.71 . The linear mixed-effects model for the diagnostic quality ratings showed a significant reader effect. Compared with the first reader, the second reader's scores were lower by 0.35 points (95\% CI, $0.28-0.42$ ).

\section{Quantitative Results}

Comparative ratios were calculated between germinal matrix/air, periventricular layer/air, subplate layer/air, and cortical layer/air for each fetal MR imaging scan. The median ratios were higher on the $3 \mathrm{~T}$ scanner than on the $1.5 \mathrm{~T}$ scanner (Table 3 ). Across all ROIs, compared with the $1.5 \mathrm{~T}$ magnet, the signal-intensity ratios from the $3 \mathrm{~T}$ magnet were higher by a median factor of 8.38 (95\% CI, 4.73-14.82) (Fig 4).

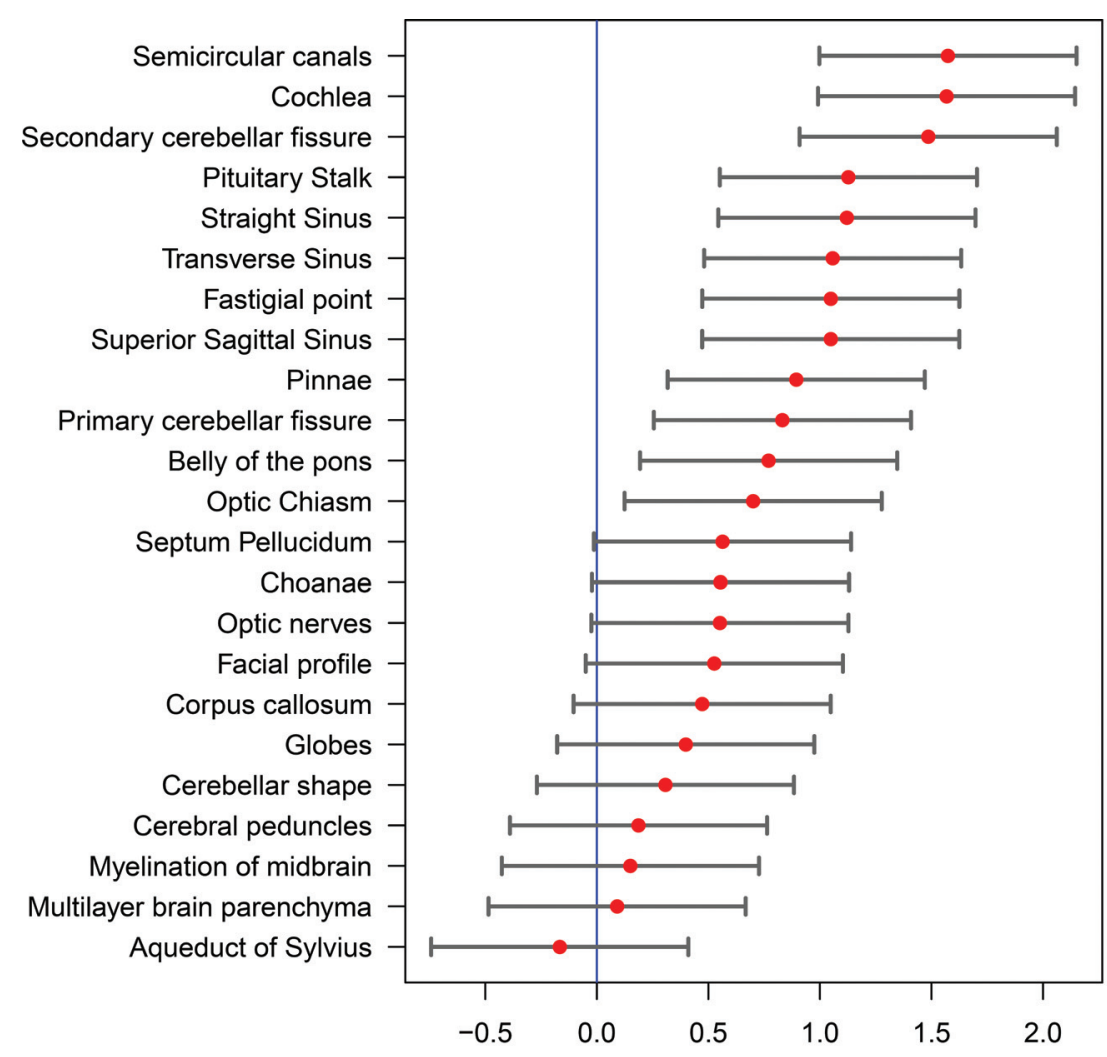

3.0T - 1.5T (confidence intervals adjusted for multiple testing)

FIG 3. Estimated differences in the mean score between magnet types at each location from a linear mixed-effects model. Estimates are accompanied by $95 \%$ confidence intervals, adjusted for multiple testing.

\section{DISCUSSION}

MR imaging is a noninvasive technique increasingly used to scan pregnant patients. Imaging performed with higher strength magnets has been proven superior in clinical practice ${ }^{3-6}$ due to their high signal intensity, resolution, reduced scanning times, and overall improved diagnostic ability. Imaging of the fetus has been evaluated with 3T, but studies were mainly focused on body imaging and not the brain and head anatomy. ${ }^{8}$ Our aim was to demonstrate the difference in spatial resolution between 1.5 and 3T magnets in fetal brain anatomy. Fetal MR images were selected between 20 and 24 weeks of gestation to ensure a similar fetal sulcation age and anatomy. ${ }^{14}$ This choice has resulted in a relatively homogeneous sample for our trial, with similar gyration patterns. In addition, the early second trimester is a crucial time in pregnancy development, counseling, and decision-making. The second trimester feMRI has been demonstrated to be safe and helpful for the diagnosis of brain anomalies when there is a sonography concern, adding valuable information and changing management in approximately $30 \%$ of cases. ${ }^{15}$ 
The classification into 4 categories for each structure was carefully considered to be a good representation of the variety of qualitative imaging acquired on the scans and also an acceptable pathway for agreement between readers. The locations selected in our study are an extensive representation of the neuroanatomy in the fetus and key structures in the assessment of anomalies. The results of the analyzed brain structures demonstrated higher values on $3 \mathrm{~T}$ magnets than on the $1.5 \mathrm{~T}$ magnets, meaning better qualitative assessment with a stronger magnetic field. In particular, all the structures involving the auditory system showed exponential statistical significance (semicircular canals, cochleae, and pinnae). Inner ear structures have already been shown to have higher resolution on $3 \mathrm{~T}$ than on $1.5 \mathrm{~T}$ magnets in healthy adult volunteers. ${ }^{16}$ In addition, the $3 \mathrm{~T}$ magnet is often chosen as the preferred technique for volumetric assessment of adult inner ear structures, ${ }^{17}$ particularly in the quantification of volumes for inner ear pathologies. The observation from the assessment with our grading also confirms that the stronger field can be used as a tool in

Table 3: Median IQR signal intensity ratio by location and magnet

\begin{tabular}{lcc}
\hline \multicolumn{1}{c}{ Location } & $1.5 T$ & 3T \\
\hline Germinal & $7.3(4.8-19.4)$ & $72.3(49.5-100.6)$ \\
Periventricular layer & $10.7(6.2-21.1)$ & $103.8(54.9-181.7)$ \\
Subplate & $12.0(8.4-25.4)$ & $165.2(97.2-270.1)$ \\
Cortical & $9.7(5.5-19.5)$ & $90.1(61.7-119.4)$ \\
\hline
\end{tabular}

Note:- $-\mathrm{QR}$ indicates interquartile range.

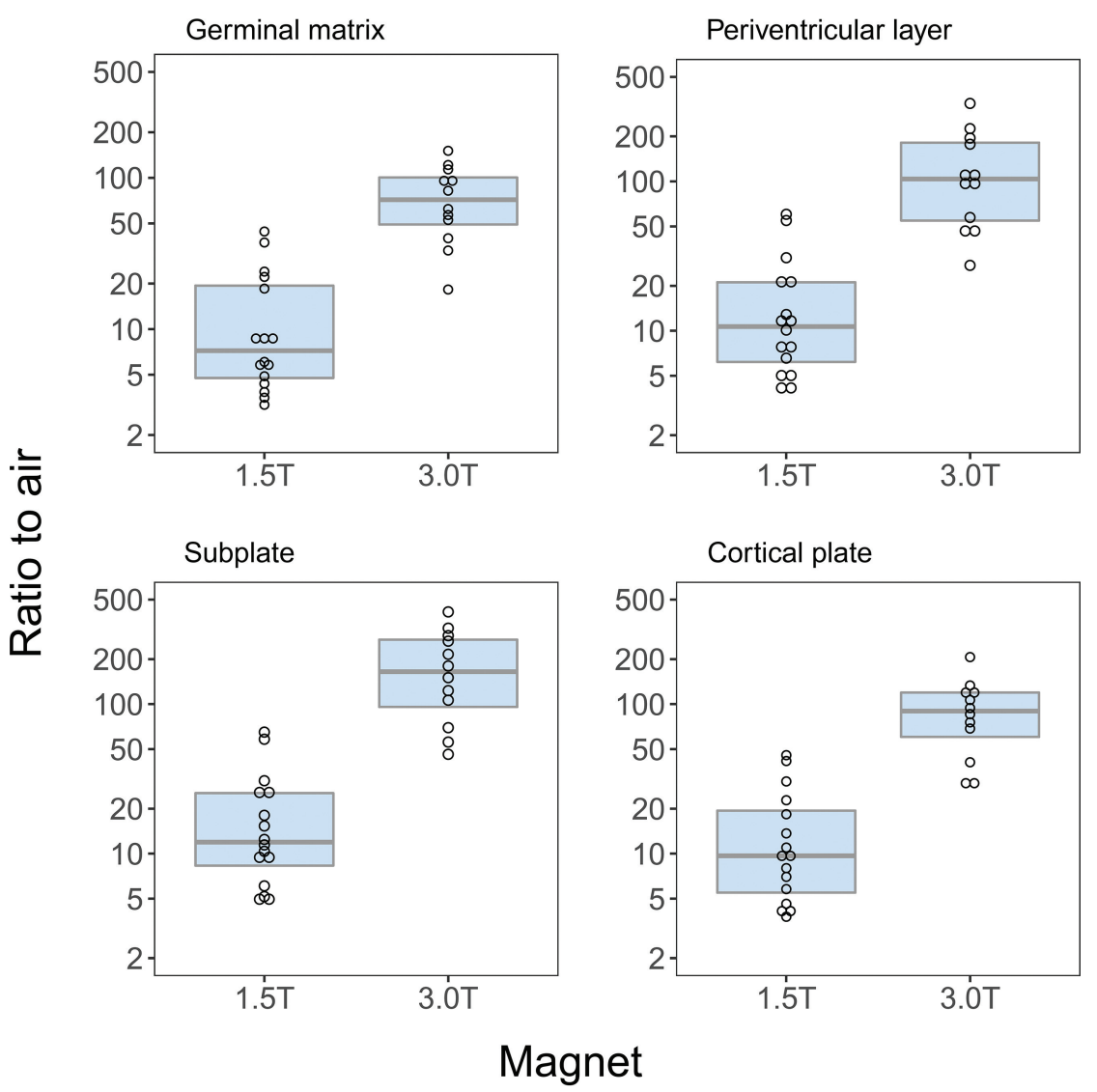

FIG 4. The ratio between signal intensity and air at each location for each magnet. Each estimated ratio is shown as a small circle. Each superimposed box indicates the 25th percentile (lower part of the box), median (heavy horizontal line), and 75th percentile (upper part of the box). Note that the vertical axis has a logarithmic scale. prenatal diagnosis to reassure normal anatomic development of the major structures of the inner ear.

On the other hand, the optic chiasm and many of the posterior fossa structures such as the belly of the pons, fastigial point, and primary and secondary fissures of the vermis revealed statistically significant differences in the qualitative assessment. Because a normal biometry of posterior fossa structures rules out many of the concerning anomalies in neurofetal imaging, it is essential to appropriately visualize and assess these structures. These structures are often critical and an area of challenge when performing neurofetal sonography; therefore, the feMRI becomes a tool of trustworthiness. ${ }^{18}$

The flow void of cerebral venous vessels, including the superior sagittal, transverse, and straight venous sinuses, also revealed better resolution on the $3 \mathrm{~T}$ magnet than on the $1.5 \mathrm{~T}$ magnet with statistical significance. Anomalies of the cerebral venous sinus in the fetus are very rare but may present as congenital anomalies, such as the persistence of the falcine sinus or agenesis of the straight sinus, or as acquired disorders, most commonly a thrombotic occlusion. ${ }^{19}$ Prenatal sonography is an effective method for diagnosing and monitoring thrombosis, but feMRI serves as a complementary technique to evaluate the full extent of the cerebral venous sinuses and extension of thrombus and to rule out cerebral parenchymal lesions secondary to hypoperfusion of the associated malformation. ${ }^{20}$

The remainder of the evaluated structures did not show statistically significant values, but the multilayering appearance of the brain on a $3 \mathrm{~T}$ magnet demonstrated higher comparative quantitative ratios than on the $1.5 \mathrm{~T}$ magnet. These quantitative differences in the signal intensity of the brain layers has been demonstrated to reflect different histologic patterns. ${ }^{21}$ The visualization of a normal transient laminar organization in the fetal brain is a relevant finding related to the normal development of the white matter in the neonate. ${ }^{21}$ Detection and characterization of malformation of cortical development have already been demonstrated to be better with a $3 \mathrm{~T}$ than with a $1.5 \mathrm{~T}$ magnet. ${ }^{22}$ The lack of statistical significance in our study group might be because only fetal studies with normal findings were included in this trial. Future studies with a combination of normal and pathologic fetal cases might enhance differences in the multilayered appearance of the fetal brain and could demonstrate the better image resolution of stronger magnets when pathology is present.

FeMRI has shown no reproducible harmful effects on pregnant women and their fetuses at a magnetic field strength of $\leq 3 \mathrm{~T} .^{7,8}$ The major concern of feMRI is thermal exposure to the mother and 
fetus because of the potential biologic damage. In fact, the main reason to avoid feMRI during the first trimester is the thermal risk. The heating caused by the radiofrequency energy is measured by the specific absorption rate (SAR), which is fixed by the appropriate authorities. In the United States, the Food and Drug Administration mandates that the SAR not exceed $4 \mathrm{~W} / \mathrm{kg}$ of the mother's body weight for all magnet strengths. ${ }^{7}$ The SAR is also limited by manufacturers to ensure that the increase in body temperature is $<0.5^{\circ} \mathrm{C}$. Experimental evidence proposes that SAR deposition to the fetus in utero is higher at $3 \mathrm{~T}^{4,6,7}$ but remains within accepted limits for clinical practice. There are recommendations to reduce the fetal SAR between 2 and 3 times with circularly polarized B1 fields instead of linear-horizontal polarization mode 2-port radiofrequency shimming. ${ }^{23}$ The ability to decrease magnet time with faster sequences at $3 \mathrm{~T}$ also has the potential to decrease the SAR. In addition, the acoustic effect has also been raised as a hypothetic concern, but the literature has shown it to be a theoretic risk rather than a real practical issue. $^{4-7}$

The advantages of a stronger magnet are primarily an increase in the signal-to-noise ratio, ${ }^{2,5}$ meaning a higher image quality, with an increased spatial-temporal resolution and a decreased acquisition time per sequence. The principal disadvantage is artifacts from a higher magnet strength (On-line Figure) (ie, susceptibility and magnetic field heterogeneity $)^{2}$ because there is stronger radiofrequency penetration, which results in more unpaired hydrogen proton spin-up.

Limitations of this study include the small sample size, the retrospective design, and the lack of prospective follow-up to ensure that each fetus is entirely neurologically normal in childhood. Another limitation is the difference between the technique and parameters applied on the $3 \mathrm{~T}$ and $1.5 \mathrm{~T}$ magnets, including the difference in section thickness between the $1.5 \mathrm{~T}$ magnet $(4 \mathrm{~mm})$ and the $3 \mathrm{~T}$ magnet $(3 \mathrm{~mm})$, particularly important in the assessment of small structures. The combination of T2-weighted single-shot FSE and steady-state FIESTA was used on the $1.5 \mathrm{~T}$ magnet compared with single T2-weighted single-shot FSE on the $3 \mathrm{~T}$ magnet. The 3D and 2D FIESTA sequences have been useful in the assessment of brain and body abnormalities in the second trimester, primarily because the FIESTA sequence provides better motion artifact-free imaging. ${ }^{24,25}$ Motion artifacts of the images were also eliminated from the sample by selectively including the best sequence from each study in the 3 different planes.

\section{CONCLUSIONS}

Both magnet strengths can examine brain and facial anatomic structures for diagnostic purposes. However, our research demonstrates a better qualitative and quantitative resolution of the evaluated structures and images with the $3 \mathrm{~T}$ magnet. This seems especially noticeable in the posterior fossa structures, venous system, and inner ears. Future studies comparing normal and abnormal fetal brains at different gestational ages may enhance the practical impact of using higher strength magnets as long as the safety of the fetus and mother are guaranteed or assured.

\section{ACKNOWLEDGMENTS}

We thank Cassandra Kapoor for administrative and editorial support and Barry Smith, MRT, for the collaboration with the MR imaging studies.

\section{REFERENCES}

1. Smith FW, Adam AH, Phillips WD. NMR imaging in pregnancy. Lancet 1983;1:61-62 Medline

2. Levine D, Barnes PD, Sher S, et al. Fetal fast MR imaging: reproducibility, technical quality, and conspicuity of anatomy. Radiology 1998;206:549-54 CrossRef Medline

3. Mack A, Wolff R, Scheib S, et al. Analyzing 3-Tesla magnetic resonance imaging units for implementation in radiosurgery. J Neurosurg 2005;102(suppl):158-64 CrossRef Medline

4. De Marzi R, Seppi K, Högl B, et al. Loss of dorsolateral nigral hyperintensity on 3.0 Tesla susceptibility-weighted imaging in idiopathic rapid eye movement sleep behavior disorder. Ann Neurol 2016;79: 1026-30 CrossRef Medline

5. Peng L, Xiao Y, Liu L, et al. Evaluation of cochlear nerve diameter and cross-sectional area in ANSD patients by 3.0-Tesla MRI. Acta Otolaryngol 2016;136:792-99 CrossRef Medline

6. Schwarz ST, Afzal M, Morgan PS, et al. The 'swallow tail' appearance of the healthy nigrosome: a new accurate test of Parkinson's disease-a case-control and retrospective cross-sectional MRI study at 3T. PLoS One 2014;9:e93814 CrossRef Medline

7. Victoria T, Jaramillo D, Roberts T, et al. Fetal magnetic resonance imaging: jumping from 1.5 to $3 \mathrm{Tesla}$ (preliminary experience). $P e$ diatr Radiol 2014;44:376-86; quiz 373-75 CrossRef Medline

8. Victoria T, Johnson A, Edgar J, et al. Comparison between $1.5 \mathrm{~T}$ and 3 T MRI for fetal imaging: is there an advantage to imaging with a higher field strength? AJR Am J Reontgenol 2016;206:195-201 CrossRef Medline

9. Krishnamurthy U, Neelavalli J, Mody S, et al. MR imaging of the fetal brain at $1.5 \mathrm{~T}$ and $3.0 \mathrm{~T}$ field strengths: comparing specific absorption rate (SAR) and image quality. J Perinat Med 2015;43:209-20 CrossRef Medline

10. Qiu Al, Mori S, Miller MI. Diffusion tensor imaging for understanding brain development in early life. Annu Rev Psychol 2015;66: 853-76 CrossRef Medline

11. Cetin I, Barberis B, Brusati V, et al. Lactate detection in the brain of growth-restricted fetuses with magnetic resonance spectroscopy. Am J Obstet Gynecol 2011;205:350.e1-7 CrossRef

12. Glenn OA. MR imaging of the fetal brain. Pediatr Radiol 2009;40: 68-81 CrossRef

13. Hothorn T, Bretz F, Westfall P. Simultaneous inference in general parametric models. Biom J 2008;50:346-63 CrossRef Medline

14. Garel C, Chantrel E, Brisse H, et al. Fetal cerebral cortex: normal gestational landmarks identified using prenatal MR imaging. AJNR Am J Neuroradiol 2001;22:184-89 Medline

15. Rossi AC, Prefumo F. Additional value of fetal magnetic resonance imaging in the prenatal diagnosis of central nervous system anomalies: a systematic review of the literature. Ultrasound Obstet Gynecol 2014;44:388-93 CrossRef Medline

16. Graf H, Schick F, Claussen CD, et al. MR visualization of the inner ear structures: comparison of 1.5 Tesla and 3 Tesla images. Rofo 2004;176:17-20 CrossRef Medline

17. Inui $\mathrm{H}$, Sakamoto $\mathrm{T}$, Ito $\mathrm{T}$, et al. Volumetric measurements of the inner ear in patients with Meniere's disease using three-dimensional magnetic resonance imaging. Acta Otolaryngol 2016;136: 888-93 CrossRef Medline

18. Katorza E, Bertucci E, Perlman S, et al. Development of the fetal vermis: new biometry reference data and comparison of 3 diagnostic modalities: 3D ultrasound, 2D ultrasound, and MR imaging. AJNR Am J Neuroradiol 2016;37:1359-66 CrossRef Medline

19. Komiyama M, Ishiguro $T$, Kitano $S$, et al. Serial antenatal sonographic observation of cerebral dural sinus malformation. AJNR Am J Neuroradiol 2004;25:1446-48 Medline 
20. McInnes M, Fong K, Grin A, et al. Malformations of the fetal dural sinuses. Can J Neurol Sci 2009;36:72-77 CrossRef Medline

21. Widjaja E, Geibprasert S, Mahmoodabadi SZ, et al. Alteration of human fetal subplate layer and intermediate zone during normal development on MR and diffusion tensor imaging. AJNR Am J Neuroradiol 2010;31:1091-99 CrossRef Medline

22. Mellerio C, Labeyrie MA, Chassoux F, et al. 3T MRI improves the detection of transmantle sign in type 2 focal cortical dysplasia. Epilepsia 2014;55:117-22 CrossRef Medline

23. Murbach M, Neufeld E, Samaras T, et al. Pregnant women models analyzed for RF exposure and temperature increase in 3T RF shimmed birdcages. Magn Reson Med 2017;77:2048-56 CrossRef Medline

24. Shen SH, Guo WY, Hung JH. Two-dimensional fast imaging employing steady-state acquisition (FIESTA) cine acquisition of fetal non-central nervous system abnormalities. J Magn Reson Imaging 2007;26:672-77 CrossRef Medline

25. Griffiths PD, Jarvis D, McQuillan $\mathrm{H}$, et al. MRI of the foetal brain using a rapid 3D steady-state sequence. Br J Radiol 2013;86:20130168 CrossRef 